\title{
Anticuerpo monoclonal anti-inmunoglobulina $E$ en el tratamiento de la rinitis alérgica
}

\section{Anti-immunoblobulin E monoclonal antibody in the treatment of allergic rhinitis}

\author{
Alfredo Naser $\mathbf{G}^{1}$, Grettel Martelo P².
}

\begin{abstract}
RESUMEN
La rinitis alérgica $(R A)$ es una enfermedad con alta incidencia a nivel mundial, sus síntomas están directamente relacionados con la exposición a un alérgeno ambiental que desencadena una cascada inflamatoria mediada por inmunoglobulina E. El tratamiento convencional actual de la RA consta del control ambiental, farmacoterapia $e$ inmunoterapia; la reciente aparición de los anticuerpos monoclonales para el manejo de las enfermedades alérgicas parece ser prometedora. Estudios han demostrado que el Omalizumab, un anticuerpo monoclonal humanizado anti IgE, es capaz de modificar la respuesta inflamatoria dependiente de IgE, constituyéndose en una posible alternativa al manejo de la $R A$.
\end{abstract}

Palabras claves: Rinitis alérgica, anticuerpo monoclonal Anti-IgE, Omalizumab.

\begin{abstract}
Allergic rhinitis $(A R)$ is a disease with global high prevalence, its symptoms are directly related to environmental exposure to an allergen that triggers an inflammatory cascade mediated by Immunoglobulin $E$. The current standard treatment of AR consists of environmental control, pharmacotherapy and immunotherapy; the recent emergence of Monoclonal Antibodies for the management of allergic diseases appears promising. Studies have shown that Omalizumab, an anti-IgE humanized monoclonal antibody, is capable of modify the inflammatory IgE-dependent response, becoming a possible alternative to the management of $A R$.
\end{abstract}

Key words: Allergic Rhinitis, Anti-IgE Monoclonal Antibody, Omalizumab.

${ }^{1}$ Médico Otorrinolaringólogo. Hospital Clínico Universidad de Chile.

${ }^{2}$ Médico. Alumna en Estadía de Capacitación en Otorrinolaringología. Hospital Clínico Universidad de Chile. 


\section{INTRODUCCIÓN}

La rinitis alérgica $(\mathrm{RA})$ representa un problema de salud mundial, con una incidencia entre $10 \%$ y $25 \%$ de la población, y una prevalencia en aumento; afecta principalmente a niños en edad escolar y adultos en edad productiva, impactando en el desempeño escolar y la productividad laboral, alterando su calidad de vida y generando costos indirectos ${ }^{1}$.

En Estados Unidos afecta aproximadamente 20 a 40 millones de personas, con un costo anual directo asociado que asciende a un billón de dólares en gastos de salud, y un costo indirecto que excede varios billones de dólares ${ }^{1}$.

La RA se define clínicamente como un desorden sintomático de la nariz, inducido por la exposición a un alérgeno y la inflamación de la mucosa nasal mediada por inmunoglobulina $E$ ( $\mathrm{lgE})$. En individuos susceptibles los alérgenos ambientales estimulan los receptores lgE de los mastocitos y basófilos generando una cascada de degranulación celular, con síntesis y liberación de mediadores inflamatorios ${ }^{2,3}$.

Tradicionalmente la RA se ha clasificado en estacional (RAE) y perenne (RAP), pero en la actualidad se emplea la clasificación propuesta por las Guías ARIA 2008 que se basan en la frecuencia de los síntomas (intermitente 0 persistente) y en la severidad de los mismos (leve o moderada-severa) ${ }^{1}$.

El tratamiento de la RA está constituido por las medidas ambientales, farmacoterapia e inmunoterapia. Las medidas ambientales consisten en evitar el contacto con el alérgeno, lo que en la práctica es muy difícil. Los antihistamínicos y los corticoides intranasales son la piedra angular del tratamiento farmacológico con una eficacia demostrada y sin mayores efectos adversos; sin embargo, requieren administración constante y de por vida. La inmunoterapia representa el único tratamiento curativo, pero requiere de mucho tiempo, es costosa, incómoda, y tiene riesgo de anafilaxia ${ }^{1,4}$.

\section{MECANISMO INMUNE DE LA RINITIS ALÉRGICA Y ROL DE LA INMUNOGLOBULINA E}

La inmupatogénesis de la RA incluye una respuesta de hipersensibilidad temprana y una tardía, mediadas por $\lg \mathrm{E}^{1,2}$.
La respuesta temprana ocurre en individuos sensibilizados a los pocos minutos de la reexposición al alérgeno. El alérgeno unido a la célula presentadora de antígeno es presentado al linfocito T-helper, el cual es activado, libera citoquinas (IL-4, IL-5, IL-3) e interactúa con linfocitos $B$ induciendo la producción de IgE específica, la cual se une al receptor de alta afinidad para IgE de los mastocitos. Esta unión induce la degranulación de los mastocitos y esto a su vez, la liberación de mediadores (histamina, leucotrienos y prostaglandinas) responsables de los síntomas de la RA (rinorrea, estornudos, prurito y obstrucción nasal) $)^{1,2}$.

La fase tardía que ocurre 4-8 horas después de la estimulación del alérgeno, se caracteriza por la presencia de linfocitos $T$, neutrófilos, basófilos y eosinófilos, los cuales liberan mediadores inflamatorios y citoquinas, perpetuando la respuesta inflamatoria ${ }^{1,2}$.

Existen dos tipos de receptores para la lgE: alta afinidad (Fc\&RI), presentes en mastocitos, basófilos, monocitos, eosinófilos y células de Langerhans; y los de baja afinidad (FceRII/CD23), implicados en funciones de presentación de antígeno (FcعRII de linfocitos B) y en la regulación de la síntesis de $\lg E^{3}$.

La IgE puede modular el grado de expresión de sus propios receptores, de manera que a mayor concentración de $\lg \mathrm{E}$, aumenta la densidad de sus receptores y la reactividad de los mastocitos y basófilos, que disminuyen con la reducción de sus concentraciones. Cuando se une la IgE al FceRI se forma una interacción entre el dominio C $3 \varepsilon$ de la $\lg$ E y la cadena alfa del receptor de alta afinidad 3 .

\section{ANTICUERPOS MONOCLONALES (MABS)}

Los mAbs son proteínas obtenidas de clones de linfocitos B, los cuales producen un anticuerpo específico que sólo reacciona con un antígeno también específico ${ }^{5,6}$.

Los mAbs se producen al aislar clones de linfocitos B; sin embargo, los cultivos de linfocitos B sólo pueden conservarse pocos días en condiciones de laboratorio, por tanto ha sido necesario desarrollar técnicas que permitan tener cultivos permanentes de éstas células. Por ello se emplean 
células híbridas, resultantes de la unión de linfocitos $\mathrm{B}$, y células de mieloma múltiple. Estas células son llamadas hibridomas y producen anticuerpos monoclonales idénticos y específicos contra un determinado antígeno $0^{5,6}$.

Los primeros mAbs sintetizados eran de origen murino, los cuales al ser administrados a humanos eran reconocidos como extraños y desencadenaban la producción de anticuerpos contra ellos, e inducían una reacción de hipersensibilidad anulando la acción de los mismos. Para evitar este problema la tecnología en ingeniería genética ha sido usada para modificar las cadenas de los mAb, obteniéndose anticuerpos quiméricos y humanizados, no anafilactógenos, y más recientemente mAb completamente humanos o fully human ${ }^{6,7}$.

\section{OMALIZUMAB}

Es un anticuerpo monoclonal humanizado recombinante (rhuMAb-E25), 95\% humano (anticuerpo IgG1 humano), y $5 \%$ de origen murino. Omalizumab se une al tercer dominio de la región constante de la cadena pesada de la lgE: el dominio C3ع (sitio de unión del receptor FceRI), bloqueando la unión de la $\lg E$ al receptor $\mathrm{FC \varepsilon RI}_{\mathrm{C}}$ de mastocitos y basófilos evitando la degranulación de los mastocitos; produce una disminución dependiente de la dosis de las concentraciones de $\lg \mathrm{E}$ y disminuye el número de receptores de los basófilos $^{8-11,14}$ (ver Figura 1).
Los inmunocomplejos Omalizumab-IgE son pequeños $(<106 \mathrm{kDa})$, no precipitantes, no fijan el complemento, no se acumulan en los glomérulos renales, ni tienen mayor riesgo de causar enfermedades inmunopatológicas ${ }^{9-11}$.

Omalizumab es el primer mAb aprobado por la FDA para el tratamiento de pacientes con asma alérgica persistente moderada-severa mayores de 12 años; con niveles elevados de IgE y test de alergias positivos para aeroalérgenos. En los pacientes asmáticos tratados con Omalizumab se ha comprobado mejoría en la calidad de vida, disminución significativa en las dosis de corticoides inhalados y de medicación de rescate, además de disminución en la frecuencia e intensidad de las exacerbaciones ${ }^{11,12}$.

\section{PRINCIPALES ESTUDIOS EN RINITIS ALÉRGICA}

En la literatura científica se encuentran múltiples estudios que evalúan Omalizumab en el tratamiento de la RA estacional y perenne, tanto en niños como en adultos ${ }^{11,13,14}$.

\section{Estudios en rinitis alérgica estacional (RAS)}

El primer gran estudio realizado con Omalizumab en

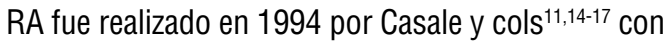
la intención de evaluar la seguridad y eficacia de dosis repetidas de este medicamento en adultos con historia de rinitis alérgica estacional (RAE) significa-

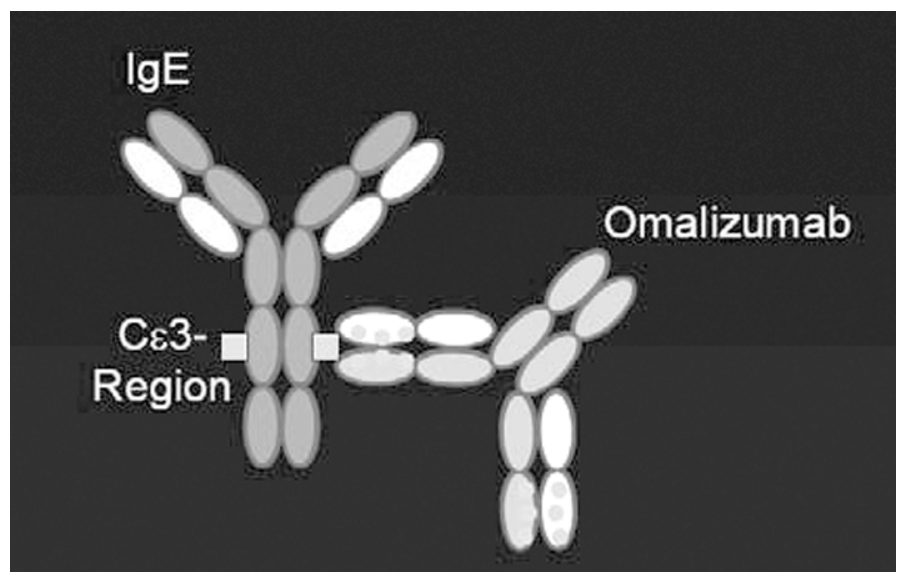

Figura 1. Omalizumab unido al dominio $\mathrm{C} 3 \varepsilon$ de la $\lg \mathrm{E}$. 
tiva. También se evaluó la relación farmacodinámica entre Omalizumab y los niveles séricos de IgE. El ensayo fue en 7 centros, doble ciego, controlado con placebo, con 240 pacientes, que fueron ramdomizados a cinco grupos: 181 pacientes recibieron una dosis de carga intravenosa de Omalizumab un mes antes del inicio de la estación, seguido por dosis adicionales en forma subcutánea (SC) 0 intravenosa (IV) dependiendo del peso corporal: $0,15 \mathrm{mg} / \mathrm{Kg} \mathrm{SC}, 0,15 \mathrm{mg} / \mathrm{Kg}$ IV ó $0,5 \mathrm{mg} / \mathrm{Kg}$ IV; Ios otros dos grupos recibieron placebo IV o SC. El medicamento fue administrado cada semana durante la estación por 12 semanas, con un seguimiento adicional de 8 semanas. Los hallazgos del estudio confirmaron que los niveles de lgE específica se correlacionaban con el puntaje de síntomas. Los sujetos tratados con Omalizumab experimentaron una reducción rápida de los niveles de lgE libre dosis-dependiente; el aclaramiento de IgE unida a Omalizumab fue más lento que el de la IgE sola, sin embargo presentaron un aumento simultáneo de los niveles de IgE total. También se observó que el Omalizumab tiene la misma distribución si es administrado IV o SC, y se confirmó su seguridad y tolerabilidad, con efectos adversos menores y similares a los del placebo. Con respecto a la eficacia, sólo 11 pacientes presentaron niveles de $\mathrm{lgE}$ indetectables; sin embargo, el hallazgo de que el puntaje de síntomas se correlacionara con el nivel de $\lg E$, y que la IgE libre disminuyera en forma dependiente de la dosis sugiere que Omalizumab pudiera aminorar los síntomas de la rinitis alérgica estacional cuando se administra en las dosis adecuadas y de acuerdo a los niveles basales de cada paciente.

Basados en los resultados del estudio anterior, Casale y cols ${ }^{15-17,21}$ realizaron otro gran estudio multicéntrico comparando tres dosis de Omalizumab administrado en forma SC: $50 \mathrm{mg}$, $150 \mathrm{mg}$ y $300 \mathrm{mg}$ con placebo. Se estudió la relación síntomas y dosis respuesta, calidad de vida, y reducción en el uso de los medicamentos de rescate en 536 pacientes con RAE moderada a severa de al menos 2 años de evolución. Los pacientes fueron randomizados y en forma dobleciega se les administró placebo y una de las tres dosis SC, 2 semanas antes del inicio de la estación polínica. El tratamiento se administró de acuerdo a Ios niveles basales de lgE: niveles entre 30-150 IU/ $\mathrm{ml}$ recibieron tratamiento en las semanas 0,4 y 8 , y los niveles entre 151 y $700 \mathrm{IU} / \mathrm{ml}$ en las semanas 0, 3, 6 y 9. Los pacientes fueron seguidos por 12 semanas, se evaluaron: puntajes de severidad de síntomas nasales y oculares (en escala de 0 a 3 ), el uso de medicación de rescate y cambios en la calidad de vida. El estudio demostró que la dosis óptima efectiva de Omalizumab para el tratamiento de la RAE es de $300 \mathrm{mg} \mathrm{SC}$, administrado cada 3-4 semanas basados en los niveles de IgE de cada paciente, con una reducción significativa de los síntomas nasales y oculares y mejoría de la calidad de vida; además con buena seguridad y tolerancia, y puede ser administrado en forma segura en estaciones polínicas posteriores.

Lin y cols ${ }^{16-18}$ realizaron un estudio doble ciego controlado con placebo durante 6 semanas en 24 pacientes con RAE por polen de ambrosía en los que se estudió la cinética del Omalizumab con las concentraciones séricas de IgE libre y las concentraciones de receptores FceRI en los basófilos, así como los cambios en la respuesta a provocaciones nasales específicas mediante rinomanometría acústica. Los pacientes se dividieron en dos grupos. Uno recibió Omalizumab en dosis SC de 0,016 $\mathrm{mg} / \mathrm{kg} / \mathrm{Ul} \mathrm{lg} \mathrm{I} / \mathrm{ml}$ los días 0 y $28(n=16)$ y el otro placebo $(n=8)$. Las concentraciones séricas de $\lg E$ libre decrecieron en el grupo tratado el día 3 ( $p$ $<0,001)$ y se mantuvieron los días 28 y 42 ( $p$ $<0,001)$. También hubo diferencias con respecto al grupo que recibió placebo $(p<0,001)$. Se observó además un incremento de la PD30 (dosis de alergeno que hace que el volumen nasal medido por rinomanometría acústica disminuya $30 \%$ en la provocación nasal con el alérgeno) en el grupo tratado $(p<0,001)$, con lo que iba disminuyendo la reducción de volumen en cada control semanal. No hubo diferencias significativas dentro del grupo que recibía placebo. A los 42 días del estudio, la expresión de receptores $F(\varepsilon R I$ en los basófilos era significativamente menor en el grupo tratado con respecto a su situación basal $(p<0,001)$ y con respecto al grupo que recibía placebo $(p<0,001)$. Esta inhibición fue máxima a los 14 días de tratamiento y se mantuvo durante todo el estudio.

En la literatura también aparecen publicaciones de estudios con Omalizumab realizados en Europa y Asia, encontrándose resultados similares a los hallados en Estados Unidos ${ }^{17,18}$. 
Adelroth y cols ${ }^{17,18}$ presentaron un estudio multicéntrico (14 centros de Europa) con distribución aleatoria, a doble ciego y controlado con placebo con 251 pacientes alérgicos al polen del abedul, a los que administraron placebo durante la estación polínica $(\mathrm{n}=86) 0300 \mathrm{mg} \mathrm{SC}$. de Omalizumab $(n=164)$ en las semanas 0 y 4 ( $\mathrm{si}$ IgE total $\leq 150 \mathrm{Ul} / \mathrm{ml}$ ) 0 en las semanas 0,3 y 6 (si lgE total $>150 \mathrm{UI} / \mathrm{ml})$. Se evaluó la eficacia en la puntuación de los síntomas (4 nasales y 3 oculares), el uso de medicamentos de rescate, la calidad de vida (RQLQ), las concentraciones séricas de lgE total e IgE libre y la presencia de efectos adversos y de anticuerpos contra Omalizumab. Comparando con el placebo se apreció una disminución de la gravedad de los síntomas nasales $(p<0,001$ en el grupo tratado con respecto a placebo) y de los síntomas oculares $(p=0,031)$, así como de las necesidades de antihistamínicos ( $p<0,001$ con respecto al placebo) y de la proporción de días sin medicamentos ( $p<0,001$ con respecto al placebo). Al aplicar la prueba de calidad de vida RQLQ se apreció una disminución significativa de la puntuación en cada uno de los siete puntos en el grupo tratado $(p<0,05)$ así como en la puntuación global $(p<0,001)$. En el $69 \%$ de los pacientes del grupo de tratamiento, la IgE sérica disminuyó por debajo de $25 \mathrm{UI} / \mathrm{ml}$; en ellos se observó disminución significativa de los síntomas y del uso de medicamentos de rescate $(p<0,05)$. Ningún paciente presentó anticuerpos frente a rhuMAb-E25 ni reacciones adversas significativas.

Okubo y $\operatorname{cols}^{19,20}$ realizaron un estudio ramdomizado, doble ciego controlado con placebo, en 100 pacientes japoneses con historia de RAE moderada-severa a polen de cedro japonés, en dos regiones de Japón (Tokyo y Osaka), entre octubre de 2001 y abril de 2002. Se administró Omalizumab a dosis de 150, 225, 300 ó $375 \mathrm{mg} 0$ placebo en forma SC cada 2 o 4 semanas de acuerdo a los niveles basales de IgE total y peso corporal. Fue evaluado el puntaje de síntomas nasales diarios y el uso de medicación de rescate; se encontró disminución significativa de las variables anteriores en el grupo de Omalizumab ( $p$ $<0,01)$, y disminución marcada de los niveles de IgE libre asociado a eficacia clínica en el grupo tratado. La incidencia de reacción en el sitio de punción fue mayor en el grupo de Omalizumab; el perfil de efectos adversos observados con Omalizumab fue similar al del placebo, y no se detectaron anticuerpos anti-Omalizumab. En conclusión Omalizumab es efectivo y seguro para el tratamiento de la RAE inducida por polen de cedro japonés.

\section{Estudios en rinitis alérgica perenne (RAP)}

Son escasos los estudios en RAP probablemente debido a que requieren tiempos más prolongados de tratamiento y seguimiento ${ }^{21}$.

Chervinsky y cols ${ }^{17,21-23}$ llevaron a cabo un estudio multicéntrico (en 16 centros de Estados Unidos), con distribución aleatoria, doble ciego y controlado con placebo de eficacia, seguridad y tolerancia del Omalizumab en la rinitis alérgica perenne. Estudiaron a 289 pacientes alérgicos a ácaros 0 a epitelios de perro 0 gato, y administraron por vía SC durante 16 semanas placebo ( $\mathrm{n}$ $=145$ ) u Omalizumab ( $n=144)$ cada 4 semanas, según el peso y las concentraciones de $\lg E(0,016$ $\mathrm{mg} / \mathrm{kg} / \mathrm{Ul} \mathrm{lg} \mathrm{E} / \mathrm{ml}$ ). Encontraron una mejoría significativa del grupo de Omalizumab con respecto al de placebo en la puntuación de los síntomas nasales ( $p<0,001)$; además observaron que la mejoría también era significativa en los pacientes que no habían respondido anteriormente a la inmunoterapia ( $p=0,012)$ ni a los corticoides intranasales $(p=0,022)$; también se redujo la necesidad y uso de antihistamínicos de rescate $(p$ $<0,005)$ y hubo una mejoría global en el cuestionario $R Q L Q$ de calidad de vida en rinoconjuntivitis ( $p$ $<0,001)$ así como en cada uno de sus puntos por separado ( $p<0,050$ inferior) y en la percepción de la eficacia por parte del paciente $(p=0,001)$. El tratamiento se toleró bien, con efectos adversos leves y similares al placebo. No se detectaron anticuerpos frente a Omalizumab.

Corren y $\operatorname{col}^{21,23}$ realizaron un estudio para evaluar el efecto de Omalizumab en la reactividad nasal y en la síntesis de lgE; 99 pacientes con rinitis alérgica perenne (RAP, fueron tratados con Omalizumab IV durante 26 semanas, se determinaron niveles basales de IgE total y libre al inicio y cada 2 semanas durante el estudio; los pacientes fueron expuestos a polvo de ácaro al inicio del ensayo y a las semanas 12 y 24 de tratamiento, y se les realizaron lavados nasales antes y después de la 
exposición en busca de anticuerpos específicos contra ácaros, anticuerpos secretores y mARN. Se encontró disminución de la IgE libre en 97\% a 99\%; reducción significativa de la reactividad nasal al polvo de ácaro en los días 80 y 164; disminución importante de la IgE específica en el día 168; y no se encontraron efectos sobre los anticuerpos secretores y sobre la producción de mARN. Los investigadores concluyeron que Omalizumab reduce los niveles de IgE libre y la respuesta nasal ante el alérgeno; pero no modula la síntesis nasal de lgE.

\section{Estudios en niños}

Al igual que en los adultos los estudios con Omalizumab en niños han evidenciado beneficios en reducción de los síntomas y en el uso de corticoides y medicación de rescate; además su uso combinado con inmunoterapia ha mostrado mayor eficacia que cuando se administran cada uno por separado. Con relación al perfil de seguridad es también similar al observado en los adultos ${ }^{11,21,25}$.

Baez y cols co,11,25,26 $^{10}$ ublicaron un estudio donde se evaluó el efecto combinado de la inmunoterapia específica (ITE) y Omalizumab sobre la liberación de la proteína catiónica eosinofílica (PCE), triptasa, IL-6, y IL-8 en la secreción nasal; 225 niños (6-17 años) con antecedentes de RAE inducida por polen de abedul y gramíneas se asignaron al azar en cuatro grupos: ITE de abedul o gramínea en combinación con anti-IgE o placebo. Se tomaron muestras de la secreción nasal de 53 pacientes antes del tratamiento Visita 1 (V1), estación de polen de abedul (V2) temporada de polen de gramínea (V3) y después de la estación de polínica (V4). Se observó reducción considerable de triptasa sólo en el grupo anti-IgE tratados en V2 $(P<0,05)$ y V4 $(P$ $<0,05)$ en comparación con el grupo placebo; durante la temporada polínica los pacientes con placebo mostraron un aumento de PCE en comparación con el basal (V2: 130,3 mcg/L; V3: 1134,2 $\mathrm{mcg} / \mathrm{L}, \mathrm{P}<0,005$; V4: 179,0 mcg/L, $\mathrm{P}<0,05)$, y niveles estables de triptasa, IL-6 e IL-8-. El tratamiento con anti-IgE resultó en valores estables de PCE y en una disminución significativa de triptasa en comparación con V1: V2: $-80,0 \mathrm{mcg} / \mathrm{L}$ (P $<0,05)$; V3: -56,3 mcg/L, que se mantuvo después de la temporada con V4: $-71,6 \mathrm{mcg} / \mathrm{L}(P<0,05)$.
Después de la temporada polínica, una disminución de la IL-6 se observó en ambos grupos (V4 grupo placebo: $-37,5 \mathrm{mcg} / \mathrm{L}$, grupo anti-IgE V4: $-42,9 \mathrm{mcg} / \mathrm{L}, \mathrm{P}<0,01)$. Concluyendo que la combinación de la ITE y anti-IgE previene del aumento de la PCE nasal y de la disminución de los niveles de triptasa en las secreciones nasales.

Otro estudio en niños realizado por RolinckWerninghaus y cols ${ }^{11,24,26}$ que incluyó pacientes entre 6 y 17 años con RAE a polen de abedul y pasto, analizó la asociación entre las concentraciones de lgE con la severidad de los síntomas y uso de medicación de rescate. El grupo A recibió placebo y el grupo B Omalizumab antes y durante la estación polínica. Los pacientes del grupo A con niveles basales elevados de IgE específica tenían una mayor puntuación de síntomas y necesidad de medicación comparado con los que tenían bajos niveles basales, esto no se observó en los del grupo B; en los pacientes tratados hubo correlación entre los niveles basales de IgE libre con la severidad de los síntomas y uso de medicación de rescate ( $p=0,031$ ), lo que no se observó con los niveles de lgE total. Los autores concluyeron que los niveles basales de lgE específica, y no de lgE total, se asocian con la severidad de los síntomas en niños con RAE por polen.

\section{Otros estudios}

Bachert y $\mathrm{co}^{26}$ sugieren que las concentraciones elevadas de IgE presentes en la poliposis nasal están relacionadas con la inflamación eosinofílica del tejido nasal poliposo, indicando un rol de la $\lg E$ en la patogénesis de esta enfermedad; además al igual que en el asma la evidencia revela que la enterotoxina del $S$. aureus podría actuar como un superantígeno resultando en la activación de células B y T con liberación masiva de lgE; basados en esto el tratamiento con Omalizumab podría suprimir la cascada inflamatoria mediada por IgE en la poliposis nasal severa o recurrente al igual que en el asma no atópica, similar a su actividad en las enfermedades respiratorias alérgicas.

\section{CONCLUSIONES}

El Omalizumab es un mAb anti-IgE humanizado recombinante que actúa uniéndose al dominio C3 
de la IgE, bloqueando la unión al receptor de alta afinidad; produciendo un rápido descenso dosisdependiente de los niveles de $\mathrm{lgE}$, el cual es sostenido a lo largo del tratamiento, lo que produce una mejoría en los síntomas, asociado a disminución del uso de medicación de rescate y mejora en la calidad de vida de los pacientes.

La dosis efectiva está relacionada con los niveles basales de IgE de cada paciente y su peso corporal.

Los estudios demuestran que Omalizumab constituye un medicamento seguro y bien tolerado, con pocos efectos adversos, y hasta ahora no ha producido reacciones anafilácticas ni se han encontrado anticuerpos anti-Omalizumab.

\section{BIBLIOGRAFÍA}

1. Lal L, Casale T, Stokes J. Pediatric Allergic Rhinitis: Treatment. Immunol Allergy Clin N Am 2005; 2: 283-99.

2. NouRi-AriA K. Recent Progress in Allergen Immunotherapy. Iran J Immunol 2008; 5(1): 1-24.

3. Plewako H, Arvidsson M, Petruson K et al. The Effect of Omalizumab on Nasal Allergic Inflammation. J Allergy Clin Immunol 2002; 110: 68-71.

4. Lee $\mathrm{CH}, \mathrm{Mo} J$. Recent Advances in Immunotherapy of Allergic Rhinitis. Current Allergy and Asthma Reports 2008; 8: 269-71.

5. Mateos M. Monoclonal Antibodies in Pediatrics: Use in Prevention and Treatment. Allergol et Inmunopathol 2007; 35(4): 145-50.

6. Cabrera-Navarro P. Anti-Immunoglobulin E, a Monoclonal Antibody to Treat Respiratory Disorders. Arch Bronconeumol 2006; 42(5): 241-5.

7. Aguillón J, Contreras J, Dotte A et al. Nuevas armas inmunológicas para la medicina del siglo XXI: Terapia biológica basada en el uso de anticuerpos monoclonales de última generación. Rev Méd Chile 2003; 131: 1445-53.

8. Féxil $R$, Martínez $R$, Negro JM, Ramírez $M$, MéridA C. Anti-IgE (Omalizumab) en el Tratamiento de la Rinitis Alérgica. Alergol Inmunol Clin 2004; 19: 133-9.

9. Cox L. How safe are the biologicals in treating asthma and rhinitis? Allergy, Asthma \& Clinical Immunology 2009; 5(4): 1-11.
10. Báez C, Schubert R, Kopp M, et al. Effect of Antiinmunoglobulin $E$ on Nasal Inflammation in Patients with Seasonal Allergic Rhinoconjuctivitis. Clin Exp Allergy 2004; 34: 107985.

11. Dodig S, Richter D, Cepelak I, Benko B. Anti-lge Therapy with Omalizumab in Asthma and Allergic Rhinitis. Acta Pharm 2005; 5: 123-38.

12. Rambasek T, Lang D, Kavuru M. Omalizumab: Where does it fit into current asthma management? Cleveland Clinic Journal of Medicine 2004; 71(3): 251-61.

13. Incorvaia C, Mauro M, Riariosforza G, Frati F, TarnatinI $F$, CaserinI M. Current and Future Applications of the Anti-IgE Antibody Omalizumab. Biologics: Targets \& Terapy 2008; 2(1): 7-73.

14. Berger W. Treatment of Allergic Rhinitis and other Inmunoglobulin E-mediated Diseases with Anti-inmunoglobulin E Antibody. Allergy Asthma Proc 2006; 27: 29-32.

15. CaSale T. Anti-Immunoglobulin E (Omalizumab) Therapy in Seasonal Allergic Rhinitis. Am J Respir Crit Care Med 2001; 164: 18-21.

16. Corren J, Casale tB, Lanier B, Buhl R, Holgate S, JIMÉNEZ P. Safety and Tolerability of Omalizumab. Clinical \& Experimental Allergy 2009; 39: 788-97.

17. Féxil R, Negro JM, Miralles JC. Omalizumab. A review of the New Treatment of Allergic Asthma and Seasonal Allergic Rhinitis. Allergol et Immunopathol 2002; 30(2): 94-9.

18. KaLINER M. Omalizumab and the Treatment of Allergic Rhinitis. Current Allergy and Asthma Reports 2004; 4: 237-44.

19. Okubo K, Ogino S, Nagakura T, Ishikawa T. Omalizumab is Effective and Safe in the Treatment of Japanese Cedar Pollen-induced Seasonal Allergic Rhinitis. Allergology International 2006; 55: 379-86.

20. Okubo K, Nagakura T. Anti-lge Antibody Terapy for Japanese Cedar Pollinosis: Omalizumab Update. Allergology International 2008; 57: 205-9.

21. Belliveau P. Omalizumab: A Monoclonal Anti-IgE Antibody. Medscape General Medicine 2005; 7(1): 27.

22. Chervinsky P, Casale $T$, Townley $R$, et al. Omalizumab, an Anti-IgE Antibody, in the 
Treatment of Adults and Adolescents with Perennial Allergic Rhinitis. Ann Allergy Asthma Inmunol 2003; 91: 160-7.

23. Corren J, Díaz-Sánchez D, Saxon A et al. Effects of Omalizumab, a Humanized Monoclonal AntiIgE Antibody, on Nasal Reactivity to Allergen and Local IgE Syntesis. Ann Allergy Asthma Immunol 2004; 93: 243-8.

24. Rolinck-Werninghaus, KeIL T, Koop M et al. Specific lgE Serum Concentration is Associated with Symptom Severity in Children with
Seasonal Allergic Rhinitis. Allergy 2008; 63: 1339-134.

25. Kamin W, Koop M, Erdnuess F, Schauer U, Zielen S, WaHN U. Safety of anti-lgE treatment with omalizumab in children with seasonal allergic rhinitis undergoing specific immunotherapy simultaneously. Pediatr Allergy Immunol2010; 21: 160-5.

26. Verbruggen K, Van Caumenberge P, Bachert C. Anti-lgE for the Treatment of Allergic Rhinitis $\tilde{n}$ and Eventually Nasal Polyps? Int Arch Allergy Immunol 2009; 148: 87-98.

Dirección: Dr. Alfredo Naser González

Santos Dumontt 999 Independencia. Santiago, Chile

E mail: aanaserg@gmail.com 